\title{
Efecto del Tipo de Alimentación sobre el Comportamiento Productivo, Características de la Canal y Calidad de Carne del Cerdo Criollo Negro Cajamarquino
}

\author{
Effect of the Feeding Type on Productive Performance, Carcass Characteristics \\ and Meat Quality of Black Creole Pig of Cajamarca
}

Manuel Paredes Arana ${ }^{1,2}$, Luis Vallejos Fernández ${ }^{1}$, José Mantilla Guerra ${ }^{1}$

\section{Resumen}

El estudio tuvo por objetivo evaluar el efecto de dos dietas sobre el comportamiento productivo, características de la canal y calidad de la carne del cerdo criollo negro cajamarquino. Se emplearon 32 lechones destetados, distribuidos en cuatro tratamientos con cuatro repeticiones por cada tratamiento y dos animales por repetición. La mitad de los cerdos fueron alimentados con desechos de restaurante y residuos de camal de aves y la otra mitad con un concentrado de maíz y soya. La mitad de cada grupo fueron machos castrados y la otra mitad hembras. Se llevó un registro de pesos de los cerdos. A los 120 días de engorde fueron sacrificados, determinándose las características de la canal y la calidad de carne a través del músculo Longissimus lumbar. Se encontró una interacción significativa entre alimentación x sexo en el peso vivo final y conversión alimenticia $(\mathrm{p}<0.05)$. El peso y rendimiento de la canal no estuvo influenciado por el sexo o alimento. Los cerdos alimentados con concentrado obtuvieron una mayor ganancia media diaria de peso y mejor conversión alimenticia y mayor proteína y menor contenido de ácido oleico en la carne que los cerdos alimentados con desechos de restaurantes y residuos de camal de aves.

Palabras clave: cerdo criollo; comportamiento productivo; carcasa; calidad de carne

\section{Abstract}

The aim of this study was to evaluate the effect of two diets on the productive performance, characteristics of the carcass and meat quality of the black creole pig of Cajamarca, Peru. A total of 32 weaned piglets were distributed in four treatments with four

\footnotetext{
${ }^{1}$ Departamento Académico de Ciencias Pecuarias, Facultad de Ingeniería en Ciencias Pecuarias, Universidad Nacional de Cajamarca, Perú

${ }^{2}$ E-mail:mepaunc@gmail.com,mparedes@unc.edu.pe
}

Recibido: 27 de febrero de 2017

Aceptado para publicación: 16 de junio de 2017 
replicates per treatment and two animals per replicate. Half of the pigs were fed with restaurant waste and poultry slaughterhouse waste and the other half with concentrate based on corn and soy. Half of each group were castrated males and the other half were females. Body weight of pigs was recorded. All animals were slaughtered at 120 days of fattening, determining carcass characteristics and meat quality through the Longissimus lumbar muscle. A significant interaction between feed $\mathrm{x}$ sex was found for body weight and feed conversion $(\mathrm{p}<0.05)$. The weight and performance of the carcass was not influenced by sex or food. Pigs fed with concentrate obtained higher body weight gain, better feed conversion, higher protein and lower content of oleic acid in the meat than pigs fed with restaurant waste and poultry slaughterhouse waste.

Key words: creole pig; productive performance; carcass; meat quality

\section{INTRODUCCIÓN}

E1 95.7\% de la producción de cerdos en el Perú proviene de unidades agropecuarias con piaras menores de 10 animales, siendo el cerdo criollo el principal genotipo con $67.2 \%$ de la población porcina nacional (INEI, 2012). El cerdo criollo es criado bajo condiciones extensivas y de traspatio con la finalidad de obtener carne y manteca, es alimentado con residuos de cocina, forrajes o subproductos agroindustriales, y el sacrificio y procesado, en muchos casos, se realiza en las propias casas (Santana, 1999), lo que conlleva a bajos índices productivos, con peso corporal de beneficio de $44 \mathrm{~kg}$ por animal en promedio (Ramos, 2008).

Algunos investigadores sugieren que el cerdo criollo no requiere estar bajo crianza intensiva (Barba et al., 1997); sin embargo, se siguen evaluando estrategias técnicas y económicamente viables, como ocurre con los sistemas de producción del cerdo ibérico (Paz y Hernández 1989). FEDNA (2013) sigue aportando normas de nutrición para un mejor desempeño del cerdo criollo. En un estudio en las Antillas Francesas con cerdos de Guadalupe de $90 \mathrm{~kg}$ de peso corporal y 190 días de edad, alimentados con pienso granulado, se encontró mayor grasa intramuscular en el cerdo criollo que en el mejorado y una capa de grasa dorsal de 22.4 mm de espesor (Renaudeau y Mourot, 2006). Este cerdo criollo al ser alimentado con dietas de soya y maíz alcanzaron ganancias de peso de $657 \mathrm{~g} /$ día en relación a los $200 \mathrm{~g}$ del grupo alimentado con caña de azúcar y soya (Xandé et al., 2009).

Otros estudios han demostrado que cuando el alimento es altamente fibroso, el cerdo criollo, opuesto a lo que se espera, presenta una menor digestibilidad de los nutrientes que el cerdo mejorado (Ly et al., 1997), confirmándose que los alimentos fibrosos como follajes de batata, yuca o eritrina, por el alto contenido de fibra y presencia de taninos, son los principales factores limitantes en cualquier tipo de cerdo (Régnier et al., 2013).

Razas de cerdo locales como el cerdo negro portugués o preto alentejano (Teixeira y Rodrigues, 2013) y el Chato Murciano de España (Galián et al., 2009) presentan gran cantidad de grasa intramuscular al beneficio. En el Perú, el cerdo criollo se le encuentra en todas las regiones naturales; así en la región costera de Tumbes se reportan pesos de beneficio de $35.4 \mathrm{~kg}$, rendimiento en canal de $56.2 \%$, grasa intramuscular de $24.7 \%$ y pérdidas por goteo de $5.3 \%$ a las 24 horas del sacrificio (Ramos, 2008). 
El uso de los residuos de cocina y restaurantes en la alimentación de cerdos en América Latina es una práctica arraigada en los sistemas tradicionales, especialmente en los sectores rurales y urbano marginales donde se mantienen pequeños hatos familiares (Benítez-Ortiz y Sánchez, 2001).

El presente estudio tuvo por objetivo evaluar la respuesta productiva, características de la canal y calidad de la carne del cerdo criollo negro de la provincia andina de Cajamarca frente a una dieta conteniendo maíz y soya y otra dieta empleada mayoritariamente por los criadores de cerdos criollos en Cajamarca, con base a desechos de restaurante enriquecidos con residuos de camal de aves.

\section{Materiales y Métodos}

Este trabajo se efectúo en la granja de cerdos de la Universidad Nacional de Cajamarca (UNC), a 2750 msnm, entre marzo y julio de 2010. Se utilizaron 32 lechones criollo negro cajamarquino, entre 30 y 45 días de edad, y fueron evaluados por un periodo de engorde de 120 días.

Los lechones fueron distribuidos en cuatro tratamientos, donde cada tratamiento tuvo cuatro repeticiones y cada repetición fue de dos animales: dos tratamientos con ocho lechones alimentados con desechos de restaurante y residuos de camal de aves, siendo uno con hembras (HDRRA) y el otro con machos castrados (MDRRA), y dos tratamientos con ocho lechones que recibieron alimento concentrado, siendo uno con hembras (H-C) y el otro con machos castrados (M-C).

La formulación del alimento concentrado se realizó considerando las recomendaciones nutricionales para cerdos ibéricos cruzados en crecimiento según las Normas FEDNA y las tablas de composición de ali- mentos FEDNA (2006). El cálculo efectuado indicó un alimento concentrado con un contenido nutricional (base fresca) de $89.69 \%$ de materia seca (MS), $16.63 \%$ de proteína cruda (PC), $3183 \mathrm{kcal} / \mathrm{kg}$ de energía metabolizable (EM), $5.35 \%$ de fibra, $0.92 \%$ de lisina, $0.30 \%$ de metionina, $0.89 \%$ de Ca y 0.63 de $\mathrm{P}$ disponible. El alimento concentrado tipo harina fue preparado en una mezcladora vertical Loyto, con capacidad de mezclado de $200 \mathrm{~kg}$.

Los desechos de restaurante (DR) fueron colectados diariamente a las 05:00 del restaurante universitario de la UNC, lo cual correspondía al desperdicio y alimento sobrante del día anterior. Previo al inicio del experimento se tomaron muestras de DR durante siete días consecutivos y en el Laboratorio de Control de Alimentos (LCA) de la UNC se determinó la variabilidad del contenido nutricional (base seca). Se determinó que en promedio tenían $22.47 \%$ de MS, $11.66 \%$ de PC, $8.56 \%$ de extracto etéreo (EE), $4.09 \%$ de fibra y $71.58 \%$ de extracto no nitrogenado (ENN).

Los residuos de camal de aves (RA) fueron colectados diariamente de un camal privado de la zona urbano marginal de Cajamarca. El camal sacrifica pollos Cobb 500 provenientes de granjas de un radio de $400 \mathrm{~km}$ y que habían pasado no menos de 12 $\mathrm{h}$ de privación de alimento. Los RA fueron sometidos a cocción en agua por $5 \mathrm{~min}$ de ebullición, en ollas de aluminio de $50 \mathrm{~L}$ de capacidad. Se dejaba enfriar por $30 \mathrm{~min}$ y se les escurría por $10 \mathrm{~min}$ en una olla con colador. Los RA contenían esófagos, buches con muy poco contenido e intestinos con materia alimenticia no digerida (eventualmente se encontraban otras partes del pollo, pero que fueron retiradas a fin de reducir la variabilidad en su composición). Los RA cocidos fueron analizados en el LCA-UNC, siendo su composición química (base seca) de $31.07 \%$ de MS, $58.16 \%$ de PC, $17.25 \%$ de EE, $10.29 \%$ de cenizas y $14.30 \%$ de ENN. 
La mezcla de DR y RA se estableció luego de los cálculos de formulación, considerando $18.54 \%$ de PC en base seca, en proporciones de nueve partes de DR y una parte de RA, de alimentos tal como fueron ofrecidos. Los alimentos fueron pesados en una báscula TCS Plataform Scale con una capacidad de $60 \mathrm{~kg}$ y una precisión de $\pm 0.01 \mathrm{~kg}$.

El suministro de alimento fue ad libitum . La primera ronda de alimento se ofreció diariamente a las 08:00, previo retiro y pesada de los residuos de alimento del día anterior, y la segunda ronda se ofreció a las 16:00. El alimento fue pesado previo a su reparto. El agua fue suministrada en bebederos de concreto con capacidad de $10 \mathrm{~L}$, con flujo de agua permanente.

Los controles de peso de los cerdos se efectuaron cada 30 días. Para los primeros 60 días del experimento se hizo en una balanza de plataforma E-ACCURA de $60 \mathrm{~kg}$ de capacidad y de $\pm 0.01 \mathrm{~kg}$ de precisión. Las pesadas posteriores fueron realizadas en una báscula Torrey tipo jaula con capacidad de $500 \mathrm{~kg}$ y una precisión de $\pm 0.05 \mathrm{~kg}$. Se registró el consumo de alimento en una tarjeta de alimentación por cada corral. Para el cálculo de la conversión alimenticia (CA) se utilizó la fórmula $\mathrm{CA}=$ Consumo de alimento (en MS) / Ganancia de peso corporal.

Los cerdos fueron sacrificados a los 120 días de edad en el camal municipal de Cajamarca. Los pesos de la canal caliente se determinaron a los 45 min post mortem en una balanza monorriel (Cardinal, SP-1000 LM), de $453.6 \mathrm{~kg}$ de capacidad (1000 libras), y se tomaron las medidas lineales de las canales con cinta métrica sobre la hemicanal derecha colgada. Se tomó como referencia para el estudio morfométrico de las canales el esquema reportado por Galián (2007). Además, se tomaron muestras del músculo Longissimus lumbar a partir de la media canal derecha y a nivel de la última costilla, para su análisis en el LCA de la UNC.
El despiece de las canales se realizó a las $24 \mathrm{~h}$ del sacrificio. Las piezas fueron pesadas en una balanza PCR-40 con capacidad de $40 \mathrm{~kg}$ y precisión de $\pm 5 \mathrm{~g}$. Las medidas de espesor de tocino dorsal (ETD) fueron realizadas en el camal utilizando un pie de rey digital 6 (Vogel, Alemania), a nivel de primera costilla, última costilla y extremo craneal del músculo glúteo medio (Peinado et al., 2004).

La humedad de la carne se determinó en la estufa de secado (UN750 Single Display, Imcolmédica) a $105^{\circ} \mathrm{C}$ durante $24 \mathrm{~h}$. Para la determinación de las pérdidas por goteo se siguió lo sugerido por Honikel (2008). La grasa intramuscular se determinó mediante extracción soxhlet con el analizador Grasa Analyzer KZF-06 (China). La determinación del ácido oleico se hizo según lo indicado por García-Fernández et al. (2010). La proteína de la carne se determinó por el método Kjeldahl modificado, con la unidad de digestión (Bloc Digest) para 6 plazas y el destilador Kjeldahl (Pro Nitro M). Las cenizas se determinaron en la mufla (Nabetherm, Alemania), durante $6 \mathrm{~h}$ a $550^{\circ} \mathrm{C}$.

Se utilizó un diseño completamente al azar en arreglo factorial de $2 \times 2$. Los datos fueron sometidos al análisis de varianza, $\mathrm{y}$ las medias fueron comparadas con la prueba de Tukey, utilizándose el software SAS 9.1.2. Se determinó el comportamiento productivo a través de la ganancia diaria de peso, consumo de alimento y conversión alimenticia, así como las características de la canal (peso de la canal caliente, rendimiento en canal, longitud de la canal, longitud de mano, longitud de jamón, longitud de pata, perímetro del jamón, perímetro de la caña, peso del lomo, peso del jamón, peso de la paleta, peso del costillar \pm panceta, peso de la cabeza, peso de las patitas, espesor del tocino dorsal) y las características de la carne (humedad, pérdida por goteo, grasa intramuscular, ácido oleico, proteína y cenizas). 
Resultados y Discusión

\section{Comportamiento Productivo}

Los pesos iniciales y finales de los lechones, la ganancia diaria de peso, el consumo de alimento y la conversión alimenticia del cerdo criollo negro cajamarquino se indica en el Cuadro 1. Las hembras alimentadas con DRRA tuvieron $10 \mathrm{~kg}$ más de peso corporal que los machos castrados $(\mathrm{p}<0.05)$, mientras que la respuesta fue opuesta en el caso del alimento balanceado, donde los machos castrados superaron en $12 \mathrm{~kg}$ a las hembras $(p<0.05)$. De acuerdo al sexo, las hembras alimentadas con DRRA adquirieron 7.93 $\mathrm{kg}$ más de peso corporal que las que consumieron concentrado, mientras que machos que consumieron concentrado alcanzaron $14.07 \mathrm{~kg}$ más de peso que los que consumieron DRRA. Resultados similares fueron, por lo tanto, encontrados para la ganancia diaria de peso.

El análisis estadístico del peso vivo final mostró interacción significativa entre las variables sexo y tipo de alimentación $(\mathrm{p}<0.05)$. Se corrobora que el cerdo criollo macho castrado tuvo una mejor respuesta en peso corporal a una dieta mejor balanceada que las hembras. Asimismo, las hembras, en condiciones alimenticias de desequilibrios nutricionales, pueden alcanzar un mejor peso corporal que los machos castrados (Rostagno et al., 2005).

Trujillo et al. (1996) y Santana et al. (1996) encontraron una menor ganancia diaria de peso en el cerdo criollo cubano en comparación a los resultados del cerdo criollo negro cajamarquino, aun cuando los cerdos cubanos fueron sacrificados con pesos de $73.7 \mathrm{~kg}$, posiblemente debido a la inclusión de menores niveles de fibra en la dieta de los cerdos cajamarquinos. Asimismo, Lemus et al. (2008) encuentran una ganancia $0.38 \mathrm{~kg}$ /día en cerdos Pelón Mexicano criados en zonas cálidas, la cual fue menor a la de este estudio. Por otro lado, Barlocco et al. (2000) reporta ganancias de $0.786 \mathrm{~kg}$ con alimento balanceado en cerdos criollo uruguayo o cerdo Pampa, y Diéguez et al. (1994) reporta ganancias de $526 \mathrm{~g} /$ día en cerdos criollo cubano que fueron sacrificados con pesos de $101.3 \mathrm{~kg}$ en promedio.

Los machos castrados consumieron más alimento concentrado que las hembras (1.42 vs. $1.30 \mathrm{~kg}$ de MS), aunque sin diferencias significativas. Asimismo, el consumo de DRRA fue similar entre sexos (1.38 y 1.40 $\mathrm{kg}$ de MS).

En la conversión alimenticia se observó una interacción significativa entre sexo y tipo de alimento $(p<0.05)$. Las hembras tuvieron mejor conversión alimenticia con DRRA que con concentrado, dado que necesitaron consumir $290 \mathrm{~g}$ menos de MS para obtener una ganancia de $1 \mathrm{~kg}$ de peso vivo durante el periodo de engorde de 120 días. La conversión alimenticia fue menos eficiente en machos castrados alimentados con DRRA que en los que consumieron concentrado (Cuadro 1). Estas diferencias demuestran que las hembras asimilaron mejor el DRRA y los machos el concentrado, posiblemente a diferencias y particularidades del funcionamiento digestivo y requerimientos nutricionales (Cadillo, 2008).

Diéguez et al. (1994) trabajando con cerdos criollos cubanos alimentado ad libitum con base a miel de caña y torta de soya, y Lemus et al. (2008) trabajando con cerdos Pelón Mexicano alimentados con forraje verde encontraron conversiones alimenticias menos eficientes que las del presente estudio. En cerdos Pampa uruguayos alimentados con pienso conteniendo $17 \%$ de proteína, desde 35 a $100 \mathrm{~kg}$ de peso corporal, se encontró una conversión alimenticia de 3.81 , y en cerdos criollo cruzados con Duroc, la conversión fue de 3.40 (Ciria y Garcés, 1996). 
Cuadro 1. Rendimiento productivo del cerdo criollo negro cajamarquino, según tipo de alimento y sexo

\begin{tabular}{|c|c|c|c|c|}
\hline \multirow{2}{*}{ Indicador } & \multicolumn{2}{|c|}{$\begin{array}{l}\text { Desechos de restaurante y } \\
\text { residuos de camal de aves }\end{array}$} & \multicolumn{2}{|c|}{ Alimento concentrado } \\
\hline & $\begin{array}{l}\text { Macho } \\
\text { castrado }\end{array}$ & Hembra & $\begin{array}{l}\text { Macho } \\
\text { castrado }\end{array}$ & Hembra \\
\hline Peso inicial, $\mathrm{kg}$ & $8.1 \pm 1.7$ & $9.1 \pm 1.1$ & $8.3 \pm 1.7$ & $10.0 \pm 0.9$ \\
\hline Peso final, kg & $55.5 \pm 4.4^{\mathrm{a}}$ & $65.5 \pm 3.8^{\mathrm{b}}$ & $69.6 \pm 5.3^{\mathrm{b}}$ & $57.6 \pm 3.7^{\mathrm{a}}$ \\
\hline Ganancia diaria, $\mathrm{g}$ & $395 \pm 39^{\mathbf{a}}$ & $470 \pm 33^{b}$ & $510 \pm 57^{\mathrm{b}}$ & $397 \pm 37^{\mathrm{a}}$ \\
\hline $\begin{array}{l}\text { Consumo } \\
\text { (MS/animal/día), kg }\end{array}$ & $1.38 \pm 0.11$ & $1.40 \pm 0.12$ & $1.42 \pm 0.09$ & $1.30 \pm 0.11$ \\
\hline Conversión alimenticia & $3.49 \pm 0.20^{\mathrm{b}}$ & $2.98 \pm 0.29^{\mathrm{a}}$ & $2.78 \pm 0.16^{\mathrm{a}}$ & $3.27 \pm 0.19^{\mathrm{b}}$ \\
\hline
\end{tabular}

${ }^{a, b}$ Superíndices diferentes dentro de filas indican diferencia significativa $(p<0.05)$

Cuadro 2. Características de la canal del cerdo criollo negro cajamarquino, según tipo de alimentación y sexo

\begin{tabular}{lcccc}
\hline Indicadores & DRRA $^{1}$ & Concentrado & Hembra & Macho \\
\hline Peso de canal caliente, kg & $42.4 \pm 3.2$ & $47.0 \pm 2.7$ & $44.5 \pm 3.5$ & $44.9 \pm 2.6$ \\
Rdto. en canal caliente, \% & $69.6 \pm 4.7$ & $72.4 \pm 4.1$ & $70.5 \pm 4.8$ & $71.4 \pm 3.9$ \\
Longitud de la canal, cm & $67.6 \pm 3.1$ & $71.3 \pm 2.5$ & $66.7 \pm 2.4^{\mathrm{a}}$ & $72.2 \pm 2.2^{\mathrm{b}}$ \\
Longitud de la mano, cm & $40.3 \pm 1.5$ & $42.8 \pm 1.3$ & $39.6 \pm 1.9^{\mathrm{a}}$ & $43.2 \pm 1.4^{\mathrm{b}}$ \\
Longitud de la pata, cm & $46.5 \pm 2.2$ & $47.1 \pm 2.0$ & $44.6 \pm 2.1^{\mathrm{A}}$ & $49.0 \pm 2.4^{\mathrm{B}}$ \\
Longitud de jamón, cm & $30.8 \pm 1.4$ & $31.5 \pm 1.6$ & $30.1 \pm 1.9$ & $32.2 \pm 2.2$ \\
Perímetro de jamón, cm & $64.5 \pm 2.3$ & $65.7 \pm 2.2$ & $61.5 \pm 2.6^{\mathrm{A}}$ & $68.7 \pm 1.9^{\mathrm{B}}$ \\
Perímetro de caña, cm & $13.9 \pm 1.2^{\mathrm{a}}$ & $14.7 \pm 1.4^{\mathrm{b}}$ & $13.8 \pm 1.1^{\mathrm{A}}$ & $14.8 \pm 2.3^{\mathrm{B}}$ \\
Peso del lomo, kg & $10.45 \pm 0.7$ & $11.51 \pm 0.4$ & $11.07 \pm 0.8$ & $10.90 \pm 0.3$ \\
Peso del jamón, kg & $11.32 \pm 0.9$ & $12.69 \pm 0.7$ & $12.05 \pm 1.1$ & $11.94 \pm 0.8$ \\
Peso de la paleta, kg & $7.72 \pm 0.8$ & $8.45 \pm 0.6$ & $8.02 \pm 0.7$ & $8.16 \pm 0.5$ \\
Peso del costillar \pm panceta, kg & $7.89 \pm 0.5$ & $8.67 \pm 0.4$ & $8.12 \pm 0.5$ & $8.45 \pm 0.3$ \\
Peso de la cabeza, kg & $3.58 \pm 0.2$ & $4.02 \pm 0.1$ & $3.70 \pm 0.4$ & $3.90 \pm 0.2$ \\
Peso de las patitas, kg & $0.99 \pm 0.11$ & $1.10 \pm 0.10$ & $1.05 \pm 0.08$ & $1.04 \pm 0.12$ \\
ETD 1 costilla, cm & $35.8 \pm 1.8$ & $33.5 \pm 1.7$ & $33.3 \pm 1.9$ & $36.0 \pm 1.5$ \\
ETD última costilla, cm & $18.0 \pm 1.1$ & $21.5 \pm 0.9$ & $21.4 \pm 1.3$ & $18.3 \pm 1.1$ \\
ETD en glúteo, cm & $29.6 \pm 2.2$ & $30.1 \pm 1.8$ & $30.6 \pm 2.1$ & $29.1 \pm 1.5$ \\
\hline
\end{tabular}

$a, b \circ A, B$ Superíndices diferentes indican diferencia significativa ( $p<0.05 \circ p<0.01$, respectivamente)

${ }^{1}$ DRRA: Desechos de restaurante y residuos de camal de aves 
Cuadro 3. Calidad de la carne del cerdo criollo negro cajamarquino, según tipo de alimentación y sexo

\begin{tabular}{lcccc}
\hline \multirow{2}{*}{ Indicadores } & \multicolumn{2}{c}{ Tipo de alimento } & \multicolumn{2}{c}{ Sexo } \\
\cline { 2 - 5 } & DRRA $^{1}$ & Concentrado & Hembra & Macho \\
\hline Humedad, \% & $71.73 \pm 1.8$ & $72.04 \pm 1.4$ & $71.58 \pm 1.1$ & $72.19 \pm 0.9$ \\
Pérdida por goteo, \% & $2.06 \pm 0.8$ & $1.91 \pm 0.5$ & $1.91 \pm 0.2$ & $2.06 \pm 0.5$ \\
Grasa intramuscular, \% & $10.96 \pm 1.1$ & $10.23 \pm 1.3$ & $10.41 \pm 1.2$ & $10.78 \pm 1.1$ \\
Ácido oleico, \% & $43.72 \pm 3.2^{\mathrm{A}}$ & $41.44 \pm 3.1^{\mathrm{B}}$ & $42.71 \pm 3.5$ & $42.46 \pm 2.8$ \\
Proteína, \% & $15.86 \pm 1.1^{\mathrm{a}}$ & $16.81 \pm 2.3^{\mathrm{b}}$ & $16.29 \pm 1.6$ & $15.94 \pm 1.7$ \\
Cenizas, \% & $2.93 \pm 0.9$ & $3.07 \pm 1.0$ & $3.03 \pm 0.5$ & $2.98 \pm 0.6$ \\
\hline
\end{tabular}

a,b o A,B Superíndices diferentes indican diferencia significativa $(p<0.05 \circ p<0.01$, respectivamente)

${ }^{1}$ DRRA: Desechos de restaurante y residuos de camal de aves

\section{Características de la Canal}

Las características de la canal se muestran en el Cuadro 2. El peso de la canal caliente (PCC) no se vio influenciada por el sexo o el tipo de alimento; sin embargo, se observó un elevado coeficiente de variación, situación que es propia del cerdo criollo, con gran variabilidad genética y alta consanguinidad, lo que no permite obtener lotes de animales homogéneos a la misma edad (Benítez-Ortiz y Sánchez, 2001; Hurtado, 2004), aún cuando se inicie con pesos de destete similares.

El rendimiento en canal tampoco fue influenciado por el sexo o el tipo de alimento, aunque se pudieron encontrar ligeras diferencias a favor de machos castrados alimentados con concentrado en relación a aquellos que consumieron DRRA, así como en machos castrados en relación a las hembras. El rendimiento en canal promedio $(71.0 \%)$ de este estudio fue inferior al $83.2 \%$ reportado para el cerdo Ibérico (Barba et al., 2001), posiblemente debido al peso y edad de beneficio, considerando que a mayor edad y peso se obtiene mayor rendimiento en la canal.
La longitud de la canal fue mayor en machos que en hembras ( $p<0.05$; Cuadro 2$)$, e inferior a los valores del cerdo Chato Murciano de $83.8 \pm 3.7 \mathrm{~cm}$ en crianza extensiva (Galián, 2007), aunque Pugliese et al. (2003) obtuvieron valores de $73.3 \mathrm{~cm}$, semejantes a los del presente estudio, en cerdos de la raza autóctona italiana Nero Siciliano, explotados al aire libre y con pesos vivos de $80.6 \mathrm{~kg}$. Estos resultados indican que el cerdo negro cajamarquino obtiene lomos de mayor longitud de acuerdo con una canal más larga que podrían alcanzar a mayor edad y peso, tan igual como los del cerdo Ibérico, y comparables a muchas razas comerciales.

El perímetro máximo del jamón es un factor de gran importancia en la industria porcina. Los valores obtenidos de $69.4 \pm 2.5$ $\mathrm{cm}$ para el grupo de machos castrados alimentados con concentrado; valores bastante inferiores a los obtenidos por Galián (2007) con el cerdo Chato Murciano criado en forma extensiva $(73.4 \mathrm{~cm})$. El peso relativo de los jamones en relación al peso de la canal entera de los cerdos cajamarquinos fue de $27.4,26.7,25.8$ y $27.5 \%$ para los lotes $\mathrm{H}-$ 
DRRA, H-C, M-DRRA y M-C, respectivamente. Por otro lado, Abeledo et al. (2004) reporta valores de $20.6 \%$ para el cerdo criollo cubano.

Las longitudes de la mano y de la pata, así como el perímetro de la caña fueron mayores en los machos que en las hembras ( $p<0.05$, Cuadro 2), En forma similar, el perímetro de la caña fue significativamente mayor en cerdos alimentados con concentrado que con DRRA ( $\mathrm{p}<0.05$, Cuadro 2).

\section{Calidad de la Carne}

En el Cuadro 3 se presentan los resultados de calidad de carne referidos a humedad, pérdida por goteo, grasa intramuscular, proteína y cenizas. No se encontraron diferencias significativas en humedad, pérdida de peso de la carne por goteo, grasa intramuscular y cenizas entre sexos o entre tipo de alimentación, ni en la interacción sexo $\mathrm{x}$ tipo de alimento. Por otro lado, el contenido de ácido oleico fue mayor en cerdos alimentados con DRRA $(\mathrm{p}<0.01)$, mientras que el porcentaje de proteína en la carne fue mayor en cerdos alimentados con concentrado $(p<0.05)$.

La pérdida de peso de la carne por goteo, el contenido en grasa intramuscular y el contenido de ácido oleico de la grasa que recubre el músculo Longissimus lumbar fue similar a la hallada por Galián (2007) en carne de cerdo Chato Murciano, mientras que Ramos (2008) reporta 5.3\% de pérdida para el cerdo criollo peruano de la región de Tumbes.

\section{Conclusiones}

- Los cerdos alimentados con concentrado obtuvieron una mayor ganancia media diaria de peso y mejor conversión alimenticia y mayor proteína y menor contenido de ácido oleico en la carne que los cerdos alimentados con desechos de restaurantes y residuos de camal de aves.
- Los machos presentaron una mayor ganancia media diaria de peso y las hembras presentaron una mejor conversión alimenticia.

- Se encontró una interacción significativa entre sexo y tipo de alimento en la conversión alimenticia $(\mathrm{p}<0.05)$. Las hembras tuvieron mejor conversión alimenticia con DRRA que con concentrado, mientras que en machos mostraron una mejor conversión alimenticia con el concentrado.

- Los machos castrados tuvieron mayores longitudes de canal, de mano y de pata, así como mayor perímetro de jamón y caña.

- El tipo de alimento no hizo variar las características de la canal, y la calidad de carne no se vio influenciada por el sexo del cerdo.

\section{Literatura Citada}

1. Abeledo C, Santana C, Pérez I, Brache, F. 2004. Rasgos de comportamiento y canal de cerdos criollo y CC2 1 alimentados con palmiche como única fuente de energía. Rev Comput Prod Porcina 11(2): 96-100.

2. Barlocco N, Vadell A, Franco J. 2000. Comportamiento productivo en el engorde de cerdos Pampa y sus cruzas con Duroc y Large White. En: XVI Reunión Latinoamericana de Producción Animal. Montevideo, Uruguay.

3. Barba C, Velázquez F, Pérez-Fernández J, Delgado JV. 1997. La sostenibilidad del cerdo negro criollo cubano dentro del desarrollo integral de la montaña. Mem I Congreso de la Sociedad Española para los Recursos Genéticos Animales. Córdova. España.

4. Barba C, Delgado J, Diéguez E, Cañuelo P. 2001. Caracterización productiva de las variedades del cerdo ibérico basada en el comportamiento en matadero. Arch Zootec 50: 125-132 
5. Benítez-Ortiz W, Sánchez D. 2001. Los cerdos locales en los sistemas tradicionales de producción. Estudio FAO Producción y Sanidad Animal 148. Roma: FAO. $211 \mathrm{p}$.

6. Cadillo J. 2008. Producción de porcinos. Lima: Juan Gutemberg Ed. 512 p.

7. Ciriá J, Garcés C. 1996. El cebo intensivo en ganado porcino. En: Buxadé C (ed). Zootecnia. Bases de producción animal, Tomo VI. Porcinicultura intensiva y extensiva. Madrid: Mundi-Prensa. p 183-197.

8. Diéguez FJ, Ly J, Pérez I. 1994. Crecimiento y canales de cerdos criollo y CC21 alimentados con miel B y soya. Rev Comp Prod Porcina 1(1): 15-21.

9. FEDNA. 2006. Necesidades nutricionales para ganado porcino. Madrid: Fundación Española para el Desarrollo de la Nutrición Animal. $55 \mathrm{p}$.

10. FEDNA. 2013. Necesidades nutricionales para ganado porcino. $2^{\mathrm{a}}$ ed. Madrid: Fundación Española para el Desarrollo de la Nutrición Animal. 109 p.

11. Galián M. 2007. Características de la canal y calidad de la carne, composición mineral y lipídica del cerdo Chato Murciano y su cruce con Ibérico. Efecto del sistema de manejo. Tesis Doctoral. Murcia, España: Univ de Murcia. 184 p.

12. Galián M, Poto A, Peinado B. 2009. Carcass and meat quality traits of the Chato Murciano pig slaughtered at different weights. Livest Sci 124: 314320. doi: 10.1016/j.livsci.2009.02.012

13. García-Martínez E, FernándezSegovia I, Fuentes-López Ana. 2010. Determinación del enranciamiento hidrolítico de un aceite de oliva mediante el grado de acidez. Departamento de Tecnología de Alimentos. Universitat Politècnica de València. $8 \mathrm{p}$.

14. Honikel KO. 1998. Reference methods for the assessment of physical characteristics of meat. Meat Sci 49: 447-457. doi: 10.1016/S0309-1740(98)00034-5
15. Hurtado E. 2004. Evaluación preliminar del cerdo criollo y los sistemas de producción en los estados Apure y Guárico de Venezuela. Tesis Doctoral. Venezuela: Universidad Central de Venezuela. $118 \mathrm{p}$.

16. [INEI] Instituto de Nacional de Estadistica e Informática 2012. IV Censo nacional agropecuario. Sistema de consulta de resultados censales. Lima-Perú. [Internet]. Disponible en: http:// censos.inei.gob.pe/cenagro/tabulados/

17. Lemus B, Hernández-Ballesteros A, Navarrete R, Rodríguez, G, de la Barrera J, Ly J. 2008. Algunos estudios de manejo de cerdos Pelón Mexicano durante el crecimiento. Rev Comp Prod Porcina 15(2): 158-162.

18. Ly J, Diéguez F, Martínez, Rarcía A. 1998. Digestion of a diet very high in fibre in Cuban Creole pigs. Anim Feed Sci Technol 72: 397-402. doi: 10.1016/ S0377-8401(97)00076-X

19. Paz A, Hernández JL. 1989. El cerdo ibérico y sus productos derivados. Madrid: Publicaciones Técnicas Alimentarias. $217 \mathrm{p}$

20. Peinado B, Poto A, Gil F, López G. 2004. Characteristics of the carcass and meat of the Chato Murciano pig. Livest Prod Sci 90: 285-292. doi: /10.1016/ j.livprodsci.2004.07.018

21. Pugliese C, Madonia G, Chiofalo V, Margiotta S, Acciaioli A, Gandini G 2003. Comparison of the performances of Nero Siciliano pigs reared indoors and outdoors. I. Growth and carcass composition. Meat Sci 65: 825-831. doi: 10.1016/S0309-1740(02)00287-5

22. Ramos D. 2008. Caracterización de la canal y la carne del cerdo criollo y de los productos cárnicos en el departamento de Tumbes-Perú. Tesis Doctoral. España: Universidad de León. 318 p.

23. Régnier C, Bocage B, Archimède $\mathrm{H}$, Noblet J, Renaudeau D. 2013. Digestive utilization of tropical foliages of cassava, sweet potatoes, wild 
cocoyam and erythrina in Creole growing pigs. Anim Feed Sci Technol 180: 44-54. doi: $10.1016 /$ j.anifeedsci.2012.12.007

24. Renaudeau D, Mourot J. 2006. A comparison of carcass and meat quality characteristics of Creole and Large White pigs slaughtered at $90 \mathrm{~kg} \mathrm{BW}$. Meat Sci 76: 165-171. doi: 10.1016/ j.meatsci.2006.10.024

25. Rostagno H, Teixeira L, Donzele J, Gomes $P$, De Oliveira $R$, Lopes $D$, Ferreira A, et al.. 2005. Tablas brasileñas para aves y cerdos. Composición de alimentos y requerimientos nutricio-nales. Brasil: Univ Federal de Vicosa. 186 p.

26. Santana I, Trujillo G, Diéguez FJ. 1996. Características de la canal de cerdos criollo, Yorkshire y L63. Rev Comp Prod Porcina 3(1): 49-53.

27. Santana I. 1999. Integración del cerdo criollo a los sistemas de explotación porcina. En: V Encuentro de Nutrición y Producción de Monogástricos. Maracay, Venezuela.

28. Teixeira A, Rodrigues S. 2013. Pork meat quality of Preto Alentejano and commercial Largewhite Landrace cross. J Integrative Agric 12: 1961-1971. doi: 10.1016/S2095-3119(13)60634-6

29. Trujillo G, Santana I, Diéguez J, Pérez I. 1996. Crecimiento de cerdos Criollo, Yorkshire y L63 alimentados con miel B y harina de soya. Rev Comp Prod Porcina 3(2): 33-39.

30. Xandé X, Mourot J, Archimède H, Gourdine J, Renaudeau D. 2009. Effect of sugarcane diets and a high fibre commercial diet on fresh meat and drycured ham quality in local Caribbean pigs. Meat Sci 82: 106-112. doi: 10.1016/ j.meatsci.2008.12.01 